\title{
The effect of intervelar veloplasty under magnification (Sommerlad's Technique) without tympanostomy on middle ear effusion in cleft palate patients
}

Mohammad Ali Hoghoughi ${ }^{1}$, Tayebeh Kazemi ${ }^{2}$, Ali Khojasteh ${ }^{3}$, Raha Habibagahi ${ }^{4}$, Zahra Kalkate ${ }^{4}$, Zeynab Zarei ${ }^{4}$, Hamidreza Hosseinpour ${ }^{5}$ and Maryam Salimi ${ }^{5^{*}}$ (D)

\begin{abstract}
Objective: Different surgical techniques and management approaches have been introduced to manage the cleft palate (CP) and its complications, such as otitis media with effusion (OME) and auditory problems. The optimal method, as well as the ideal time for palatoplasty and ventilation tube insertion, are the subject of controversy in the literature. We aimed to evaluate The Effect of Intervelar Veloplasty under Magnification (Sommerlad's Technique) without Tympanostomy on Middle Ear Effusion in Cleft Palate Patients.

Methods: non-syndromic cleft palate patients from birth to 24 months who needed primary palatoplasty from April 2017 to 2019 were enrolled in this study. intravelar veloplasty (IVP) surgery under magnification has been done by the same surgeon. Likewise, Otoscopy, Auditory Brainstem Response (ABR), and tympanometry were performed for all the patients before and six months after palatoplasty.
\end{abstract}

Results: Tympanograms were classified into two categories according to shape and middle ear pressure, and it was done in 42 children (84 ears). Type B curve was seen in 40 cases (80 ears) before surgery which reduced significantly $(P<0.005)$ to 12 cases in the left ear and 14 cases in the right ear after surgery. So, after surgery, $70 \%$ of the tympanogram of left ears and $66.6 \%$ of the tympanogram of Rt ears were in normal condition (type A tympanometry). ABR was done for 43 patients (86 ears) before surgery and six months after palatoplasty. Data were shown that 40 of the patients had mild to moderate hearing loss before surgery, which reduced significantly $(P<$ 0.005) to 9 in the left ear and 11 in the right ear after palatoplasty. So, after surgery, $79 \%$ of ABR of left ears and $73.8 \%$ of ABR of right ears were in normal status (normal hearing threshold).

Conclusions: Intervelar veloplasty under magnification (Sommerlad's technique) significantly improved the middle ear effusion without the need for tympanostomy tube insertion.

Keywords: Cleft palate, Otitis media with effusion, Tympanostomy tube, Ventilation tube

* Correspondence: salimimaryam7496@gmail.com

${ }^{5}$ Student Research Committee, Shiraz University of Medical Sciences, 71936 -

13311 Shiraz, Iran

Full list of author information is available at the end of the article

(c) The Author(s). 2021 Open Access This article is licensed under a Creative Commons Attribution 4.0 International License, which permits use, sharing, adaptation, distribution and reproduction in any medium or format, as long as you give appropriate credit to the original author(s) and the source, provide a link to the Creative Commons licence, and indicate if changes were made. The images or other third party material in this article are included in the article's Creative Commons licence, unless indicated otherwise in a credit line to the material. If material is not included in the article's Creative Commons licence and your intended use is not permitted by statutory regulation or exceeds the permitted use, you will need to obtain permission directly from the copyright holder. To view a copy of this licence, visit http://creativecommons.org/licenses/by/4.0/ The Creative Commons Public Domain Dedication waiver (http://creativecommons.org/publicdomain/zero/1.0/) applies to the data made available in this article, unless otherwise stated in a credit line to the data. 


\section{Introduction}

The orofacial cleft with the prevalence of one in 700 births is considered the most common birth anomaly [1]. Likewise, persistent eustachian tube dysfunction is thought to be the primary factor responsible for the higher rates of more serious middle ear pathology observed in children with cleft palate, such as tympanic membrane perforation, middle ear atelectasis, cholesteatoma, and otitis media with effusion (OME) [2]. Moreover, OME entangles approximately 30 to $40 \%$ of children at least once while rising to more than $90 \%$ in children with CP [3, 4].

Insertion of ventilation tubes has been considered as the optimal treatment for otitis media in patients with cleft palate, even though controversy surrounds the timing of ear tube placement. Furthermore, concerning excessive compliance with the eustachian tube, high rates of otorrhea following myringotomy and ventilation tube insertion before palatoplasty have been reported [5].

It was also shown that chronic otorrhea stopped only after palatoplasty in a few children who had early myringotomy and tube insertion [6, 7]. Likewise, no long-term research has been conducted to evaluate the effect of early placement of ventilation tubes on speech outcomes in patients born with cleft palate. Therefore, various surgical procedures have been introduced to correct the anatomic structures of patients with cleft palate along eliminate the problem.

Among different surgical techniques, the Sommerlad and Furlow palatoplasty techniques seemed to generate the best outcomes for middle ear function and speech. The intravelar veloplasty (IVVP), which was planned by Kriens and propagated by Sommerlad, based on the anteriorly malpositioned bundles dissection from the posterior edge of the hard palate followed by repositioning levator sling and palatal musculature in the midline along with radical muscle dissection as well as posterior repositioning performed under the operating microscope [8].

This paper aims to show the outcome of intravelar veloplasty (IVVP) surgery under magnification (Sommerlad's technique) on eustachian tube function and the middle ear status.

\section{Materials and methods}

\section{Study design and patient selection}

In this cross-sectional study, non-syndromic cleft palate patients from birth till 24 months needed primary palatoplasty, referring to the Cleft Lip and Palate Center of Shiraz University of Medical Sciences from April 201 to April 2019, were enrolled in this study. Patients who had anomalies of the external ear that probe insertion was not possible and had sensorineural hearing loss were excluded from the study. Likewise, the patients who suffered from extremely severe middle ear effusion under the guide of otorhinolaryngologists were referred for tympanostomy tube insertion and were excluded from our study. Intravelar veloplasty (IVVP) surgery under magnification has been done by the same surgeon for all the involved patients.

\section{Surgical technique}

Under general anesthesia in the supine position, the head was hyperextended, and a rolled towel was inserted under the shoulder. Then, we applied a mouth gag retractor for exposure. After that, lidocaine and epinephrine $(1 / 200,000)$ were injected into the hard and soft palate and waited for 7-10 min. Intervellar veloplasty (IVVP) was started with an incision between the oral and nasal layer, and we could find this junction under magnification ( $\times 2-3$, VARIO,700 Zeiss). The incision was extended enough to the posterior part of the hard palate edges properly. The oral layer was elevated from muscles and nasal layers. At this stage, the nasal layer from the uvula to the hard palate was closed with 5.0 Polydioxanone (PDS) (Fig. 1).

At this important stage, levator velli palatine and a small part of tensor velli palatine muscles were detached under magnification $(\times 4-5)$ from the nasal layer precisely and repositioned to the most posterior part of the soft palate (Fig. 2). In the end, the oral layer was closed with 4.0 PDS. If any tension of midline closure is present, the relaxing incision would be done laterally.

\section{Pre and postoperative assessment}

Auditory Brainstem Response (ABR) is an electrophysiologic assessment tool for estimating threshold, for newborn hearing screening, or for determining whether a hearing loss may be sensory, neural, or retrocochlear and was performed for all the patients before and 6

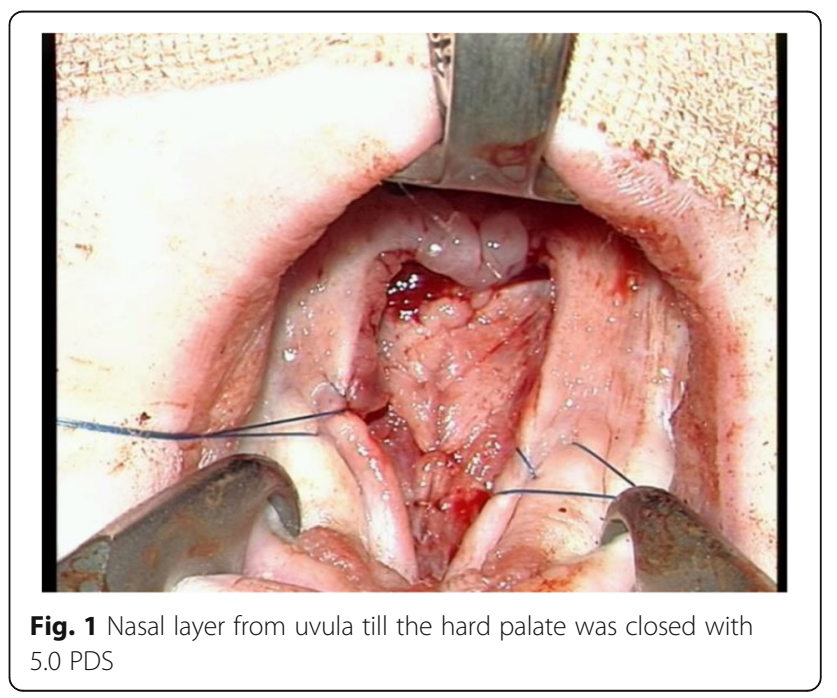




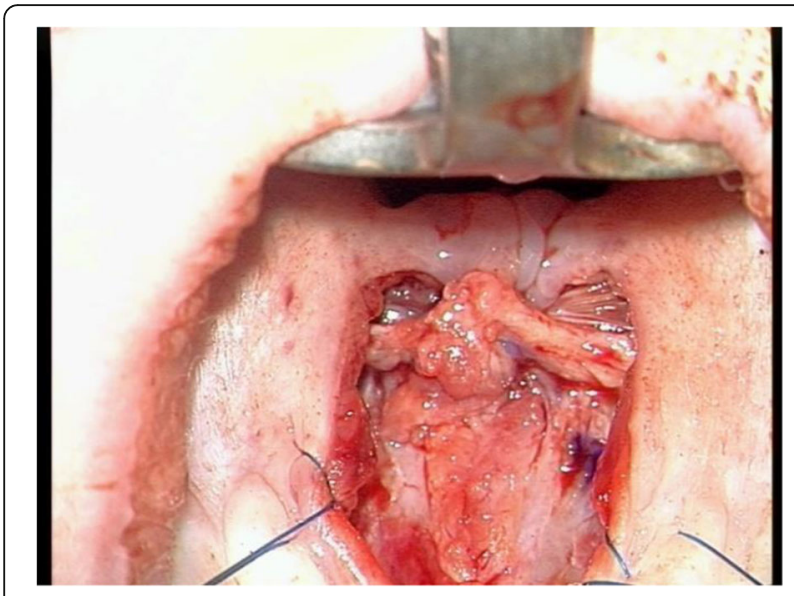

Fig. 2 Reposition of levator muscles to the most posterior part of the hard palate

months after palatoplasty. Likewise, otoscopy examination also was accomplished for all of them. The Auditory Brainstem Response (ABR) threshold detection test was performed by click stimuli from 0 to $100 \mathrm{~dB}$ HL in the frequency 1000 to $4000 \mathrm{HZ}$. The hearing assessment was done by ABR using Eclipse from Intracoustic Company. The mean hearing loss was calculated for each ear as an average of hearing levels at $0-100 \mathrm{~dB}$. The ears were classified into seven hearing level groups according to Clark classification [9], $-10-15 \mathrm{~dB}$ was considered normal hearing, $16-25 \mathrm{~dB}$ was considered a slight hearing loss, $26-40 \mathrm{~dB}$ was considered a mild hearing loss, 41-55 dB was considered moderate, 56$70 \mathrm{~dB}$ was considered moderately severe,71-90 dB was considered severe and above $90 \mathrm{~dB}$ was considered profound hearing loss.

Tympanometry, which can be used to evaluate the middle ear function, was done in all children before and six months after palatoplasty to assess the middle ear using AZ 26 tympanometer from intracystic company tympanometer with a probe frequency of $226 \mathrm{~Hz}$. Tympanograms are categorized according to the shape of the plot and the tympanometric findings were classified according to Jerger as a normal tympanogram which is labeled Type $\mathrm{A}$, that represent a normal pressure in the middle ear with normal mobility of the eardrum and ossicles and middle-ear pressure between +50 and $-99 \mathrm{mmH}^{\wedge}$ sub $2^{\wedge} \mathrm{O}$; Type $B$ for flat trace without a well-defined compliance peak, and $\mathrm{C}$ tympanograms for middle-ear pressure between -100 to $-400[\mathrm{M} 1] \mathrm{mm}-\mathrm{H}^{\wedge}$ sub $2^{\wedge} \mathrm{O} .7$, Type $B$ tympanogram may reveal fluid in the middle ear, perforation of the tympanic membrane, scarring of the tympanic membrane, lack of contact between the ossicles, or a tumor in the middle ear [10].

\section{Ethical consideration and statistical analysis}

The written informed consent was signed by the parents of the patients and the protocol of the study was approved by the local ethical committee of Shiraz University of Medical Sciences (IR.SUMS.REC.1398.535). Descriptions were given to parents about how to do the test and how to respond to the examiner. Individuals with full satisfaction participate in this research, and written consent will be signed by one of the parents.

\section{Statistical analysis}

After data collection, the information was entered into version 26 of SPSS software for statistical analysis. We used the paired T-test to analyze the tympanometric data of the patients before and after the palatoplasty. The frequencies of types B, C, and A were calculated for the total sample and the bilateral cleft lip and palate (BCLP), unilateral cleft lip and palate (UCLP), and isolated cleft palate (ICP) groups. We also merged the two types A and B for statistical results.

\section{Results}

A total of 43 patients (16 male and 27 Female) with a mean age of 12 months were included in the study. Among them, 21 patients had ICP, 17 had UCLP, 3 had BLCLP, and two had submucous clefts. The first table contains the demographic data (Table 1).

Tympanogram results were divided into two categories (A and B) according to shape and middle ear pressure, and it was done in 42 children (84 ears). Type B curve was seen in 40 cases before surgery which reduced to 12 cases in the left ear and 14 cases in the right ear after surgery. So, after surgery, $70 \%$ of the tympanogram of left ears and $66.6 \%$ of the tympanogram of right ears were in normal condition (type A tympanometry). A comparison of Tympanogram before and post-surgery in our study revealed statistically significant differences at this test $(P<0.005)$.

Table 1 Demographic data of cleft palate patients and their correlation with age

\begin{tabular}{|c|c|c|c|c|}
\hline \multirow[t]{2}{*}{ Variable } & \multirow{2}{*}{$\begin{array}{l}\text { Average } \\
S D^{\mathrm{a}} / \\
\text { frequency }\end{array}$} & \multicolumn{3}{|l|}{ Gender } \\
\hline & & Male & Female & P. value \\
\hline Age (month) & $11.5 \pm 2.1$ & $11.4 \pm 2.3$ & $11.6 \pm 2.0$ & 0.703 \\
\hline$I C P^{b}$ & 21 & 8 & 13 & 0.909 \\
\hline$U C L P^{C}$ & 17 & 6 & 11 & 0.838 \\
\hline$B L C L P^{d}$ & 3 & 2 & 1 & 0.285 \\
\hline Submucosa cleft & 2 & 0 & 2 & 0.276 \\
\hline
\end{tabular}

${ }^{\mathrm{a} S t a n d a r d ~ d e v i a t i o n}$

bIsolated cleft palate

'Unilateral cleft lip and palate

${ }^{\mathrm{d}}$ Bilateral cleft lip and palate 
ABR was done for 43 patients (86 ears) before surgery and 6 months after palatoplasty. Data were shown that 40 of the patients had mild to moderate hearing loss before surgery which reduced to 9 in the left ear and 11 in the right ear after palatoplasty. So, after surgery, $79 \%$ of left $A B R$ and $73.8 \%$ of $A B R$ of right ears were in normal status (normal hearing threshold). The difference between the two groups regarding $A B R$ in the right ear and left ear was statistically significant $(P<0.005)$.

Table 2 demonstrates the details of ABR and tympanogram before and 6 months after surgery.

About $30 \%$ of the $93 \%$ of the population who experienced moderate to severe otitis media along with effusion recovered and became normal after palatoplasty surgery which was statistically significant. Likewise, $66.67 \%$ of the patients had mild to moderate otitis media and effusion before surgery which reduced to $33.33 \%$ after palatoplasty.

\section{Discussion}

$\mathrm{CP}$ is not an isolated problem and is accompanied by many disorders such as auditory problems as well as middle ear effusion. The goals for treatment of the cleft palate patient besides normal speech, maxillofacial growth, and avoiding fistulas are eliminating the problems of middle ear infections as well as improve the hearing ability of these patients. The optimal management and intervention time of CP and each of its complications has been widely discussed and were the subject of controversy in the literature. Likewise, eustachian tube dysfunction which is considered as one of the major problems in cleft palate patients must have managed accurately not only to prevent the future OME but also for proper hearing and following speech ability. The results of our study indicated the improvement in middle ear function and hearing conditions as well as reducing the need for ventilation tube insertion in cleft patients after IVVP Under magnification.

Based on our results, the OME reduced from 95 to $32 \%, 6$ months after Sommerlad IVVP. It shows that the applied method performed significantly in the improvement of the middle ear effusion. Several studies approved the effect of palatoplasty in reducing the incidence of OME, while they did not find any statistically significant correlation between the surgical technique and the reducing impact on middle ear effusion [11-14]. On the other hand, Hassan et al. reported that Sommerlad IVVP was associated considerably with a lower incidence of OME in comparison with the (Veau-WardillKilner) VWK palatoplasty [15]. Overall with respect to the results of current and previous studies.

According to the hearing assessments by ABR, $93 \%$ had mild to moderate hearing loss before surgery which reduced significantly to $23 \%, 6$ months after Sommerlad IVVP. Antonelli et al. Carrol et al. reported the superiority effect of Furlow palatoplasty on von Langenbeck, 2-flap, and the VWK palatoplasties. Likewise, Musgrave et al. found the better result of hearing ability in von Langenbeck in comparison with VWK palatoplasty, while surgical technique was not a related factor to hearing tech auditory function in a study that was done by Lithovius et al. It is noteworthy that the study above did not include the Sommerlad IVVP technique. Therefore, a comprehensive comparison between different surgical techniques regarding the post-operation auditory function has not been possible so far, but it can be said that all palatal reconstruction methods improve hearing function.

There have been different reports in terms of the ideal time for management of OME via tympanostomy tube. Based on our data from tympanogram assessments which have been done before surgery and 6 months after surgery, a significant amount of OME was revealed 6 months after palatoplasty without the need for a tympanostomy tube. According to the parallel study, Hassan et al. believed that secretory otitis media improved 6 months after palatoplasty without the requisite ventilation tube insertion. He also reported that IVVP is a contributing factor in eustachian tube normalization function [15]. Similarly, other studies corroborated that palatal muscle reconstruction and relocation decrease the rate of eustachian tube dysfunction [16, 17]. Brgoch et al. and D'Andréa et al. emphasized the role of Sommerlad IVVP in reducing the incidence need of ventilation tube placement $[11,18]$.

Table 2 Assessment of ABR and tympanogram before and 6 months after surgery

\begin{tabular}{|c|c|c|c|}
\hline Variable & Pre-operation Number (\%) & Post-operation Number (\%) & $P$. value \\
\hline \multicolumn{4}{|c|}{ Abnormal ABR ${ }^{a}$} \\
\hline Right & $40(95.2)$ & $11(25.6)$ & $P<0.005$ \\
\hline Left & $40(95.2)$ & $9(20.9)$ & \\
\hline \multicolumn{4}{|c|}{ Abnormal Tym. ${ }^{b}$} \\
\hline Right & $40(93.0)$ & $14(33.3)$ & $P<0.005$ \\
\hline Left & $40(93.0)$ & $12(28.6)$ & \\
\hline
\end{tabular}

${ }^{a} A B R$ Auditory Brainstem Response

${ }^{\mathrm{b}}$ Tym Tympanogram 
On the contrary, Mantilla et al. suggested the combination of palatoplasty and ventilation tube insertion. $\mathrm{He}$ pointed to the potential noxious effect of repeated use of anesthetics on neurocognitive development while he did not find any significant correlation. The author also cited the association of language development with more cleft palate-related surgery. The conclusion mentioned above could be wrong since cleft palate affects speech development and patients with the more severe form of deformity logistically need more surgery. Therefore, the lower speech-related abilities were attributed to the more disease severity [19]. Likewise, Balraj et al. suggested that combining procedures may be more costeffective and decrease the total burden on families [20].

Furthermore, in vitro evidence showed the apoptosis effect of anesthesia exposure on the nervous system in rodents [21]. On the other hand, several clinical studies denied the deleterious neurodevelopmental effect of anesthetics in pediatrics $[22,23]$. To clarify the above statements, it seems that equal consideration of the number of surgeries in both combined and separate groups of surgery caused a mistake during comparing. To describe more precisely, palatoplasty and palatal muscles reconstruction decrease the recurrent incidence of OME and so the total number of tympanostomy tube insertions was reduced after 6 months of the first operation. Therefore, the total amount of anesthetic exposure, hospital stay, and the burden is expected to reduce theoretically.

It also should be considered that simultaneous procedures require collaboration and the presence of two different specialized teams. For the same reason, an uninvited interruption, suspension, and delays in scheduling the procedures are unavoidable.

From the point of view of surgical complications, ventilation tube insertion has been proven to associate with tympanic membrane perforation, otorrhea, and tympanosclerosis. The cumulative evidence at the present study indicates that the aforementioned complications will be decreased by improving the eustachian tube function by the IVVP under magnification (Sommerlad technique) and post-operation follow-up in 6 months.

Our perusal was not without limitation. The observational methodology does not allow us to demonstrate the efficacy of the used approach properly. Moreover, the possibility of selection bias should also be addressed. Likewise, the control group of combined surgery did not exist. The absence of the control group was due to ethical considerations. Since the aforementioned approach and the surgical method had the best result for the patients clinically according to the surgeon's opinion of this center, it was not possible to have a control group who underwent surgery in another way according to the ethical consideration. Furthermore, we did not include the patients with simultaneous cleft palate, and craniofacial syndromes considered the contributing factor of recurrent OME.

\section{Conclusions}

Intervelar veloplasty under magnification significantly improved the middle ear effusion for $63 \%$ and also hearing function for $70 \%$ without the need for simultaneous tympanostomy tube insertion during the 6 months after surgery. Therefore, tympanostomy tube insertion may not be necessary, at least after this technique of primary palatoplasty. However, regular follow-up is needed to evaluate the status of middle ear effusion.

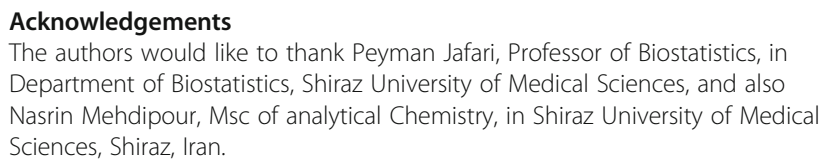

\section{Authors' contributions}

MA. H. conceived the main idea. T. K. and R. H. contributed in data gathering and analysis. A. K. and Z. K. drafted the manuscript. M. S and HR. H revised the manuscript and acted as the guarantor of the manuscript. All authors read and approved the final manuscript.

\section{Funding}

This research did not receive any specific grant from funding agencies in the public, commercial, or not-for-profit sectors.

Availability of data and materials

SPSS data of the participant can be requested from the authors. Please write to the corresponding author if you are interested in such data.

\section{Declarations}

Ethics approval and consent to participate

Written informed consent was obtained from the patients in our study. The purpose of this research was completely explained to the patients, and they were assured that their information would be kept confidential by the researcher. Participants below the age of 16 were given written informed consent from parents/legal guardians. The ethical committee approved this research of Shiraz University of Medical Sciences. All procedures performed in studies were following the ethical standards of the Shiraz University of Medical Sciences.

\section{Consent for publication}

Written informed consent was obtained from the patients regarding the publication of this study. For participants below the age 16 years old and written informed consent was given from parents/legal guardians.

\section{Competing interests}

The authors declare that they have no competing interests.

\section{Author details}

${ }^{1}$ Plastic \& Reconstructive Surgery Research Center, Shiraz University of Medical Sciences, Shiraz, Iran. ${ }^{2}$ Otolaryngology Research Center, Shiraz University of Medical Sciences, Shiraz, Iran. ${ }^{3}$ Burn and Wound Healing Research Center, Shiraz University of Medical Science, Shiraz, Iran.

${ }^{4}$ Orthodontic Research Center, Shiraz University of Medical Science, Shiraz, Iran. ${ }^{5}$ Student Research Committee, Shiraz University of Medical Sciences, 71936 - 13311 Shiraz, Iran 
Received: 5 May 2021 Accepted: 25 August 2021

Published online: 01 September 2021

\section{References}

1. Lohmander A, Olsson M. Methodology for perceptual assessment of speech in patients with cleft palate: a critical review of the literature. Cleft Palate Craniofac J. 2004:41(1):64-70.

2. Funamura JL, Said M, Lin SJ, McKinney S, Tollefson TT. Eustachian tube dysfunction in children with cleft palate: a tympanometric time-to-event analysis. Laryngoscope. 2020;130(4):1044-50.

3. Dissaux C, Grollemund B, Bodin F, Picard A, Vazquez M-P, Morand B, James I, Kauffmann I, Bruant-Rodier C. Evaluation of 5-year-old children with complete cleft lip and palate: Multicenter study. Part 2: Functional results. J Cranio Maxillofac Surg. 2016;44(2):94-103.

4. Mirashrafi F, Saedi B, Anari MR, Garmaroudi G, Toosi R, Abouhamzeh K. Evaluating the frequency of ear problems in patients with cleft lip and palate. J Iran Med Council. 2021:4:49-54.

5. Yang N, Beaudoin P-L, Nguyen M, Maillé H, Maniakas A, Saliba I. Subannular ventilation tubes in the pediatric population: clinical outcomes of over 1000 insertions. Int J Pediatr Otorhinolaryngol. 2020;131:109859.

6. Kuo C-L, Tsao Y-H, Cheng H-M, Lien C-F, Hsu C-H, Huang C-Y, Shiao A-S. Grommets for otitis media with effusion in children with cleft palate: a systematic review. Pediatrics. 2014;134(5):983-94.

7. Ponduri S, Bradley R, Ellis PE, Brookes ST, Sandy JR, Ness AR. The management of otitis media with early routine insertion of grommets in children with cleft palate—a systematic review. Cleft Palate Craniofac J. 2009:46(1):30-8.

8. Sommerlad BC. A technique for cleft palate repair. Plastic Reconstruct Surg. 2003;112(6):1542-8.

9. Clark JG. Uses and abuses of hearing loss classification. Asha. 1981;23(7): 493-500.

10. Jerger J. Clinical experience with impedance audiometry. Arch Otolaryngol. 1970;92(4):311-24.

11. D'Andréa G, Maschi C, Savoldelli C, Caci H, Bailleux S. Otologic outcomes with two different surgical protocols in patients with a cleft palate: a retrospective study. Cleft Palate Craniofac J. 2018;55(9):1289-95.

12. Güneren E, Özsoy Z, Ulay M, Eryilmaz E, Özkul H, Geary PM. A comparison of the effects of Veau-Wardill-Kilner palatoplasty and Furlow double-opposing Z-plasty operations on eustachian tube function. Cleft Palate Craniofac J. 2000;37(3):266-70

13. Spauwen PH, Goorhuis-Brouwer SM, Schutte HK. Cleft palate repair: furlow versus von Langenbeck. J Cranio Maxillofac Surg. 1992;20(1):18-20.

14. Wilson AT, Grabowski GM, Mackey WS, Steinbacher DM. Does type of cleft palate repair influence postoperative eustachian tube dysfunction? J Craniofac Surg. 2017;28(1):241-4.

15. Hassan ME, Askar S. Does palatal muscle reconstruction affect the functional outcome of cleft palate surgery? Plastic Reconstruct Surg. 2007;119(6):185965.

16. Broen PA, Moller KT, Carlstrom J, Doyle SS, Devers M, Keenan KM. Comparison of the hearing histories of children with and without cleft palate. Cleft Palate Craniofac J. 1996;33(2):127-33.

17. Huang MH, Lee $\mathrm{S}$, Rajendran K. A fresh cadaveric study of the paratubal muscles: implications for eustachian tube function in cleft palate. Plastic Reconstruct Surg. 1997;100(4):833-42.

18. Brgoch M, Dodson K, Kim T, Kim D, Trivelpiece R, Rhodes J. Timing of Tympanostomy tube placement and efficacy of Palatoplasty technique on the resolution of chronic otitis media: a cross-sectional analysis. Eplasty. 2015;15:298-302

19. Mantilla-Rivas E, Mamidi I, Rokni A, Brennan A, Rana MS, Goldrich A, Manrique M, Shah M, Reilly BK, Rogers GF. Efficacy and safety in combining primary palatoplasty and myringotomy in patients with cleft palate. Plastic Reconstruct Surg Global Open. 2020;8(5):e2824.

20. Balraj A, Kurien M, Job A. Concurrent surgeries in ENT: a cost-effective and safe approach in patients requiring multiple definitive procedures. J Laryngol Otol. 2004;118(1):31.

21. Sanders R, Hassell J, Davidson A, Robertson N, Ma D. Impact of anaesthetics and surgery on neurodevelopment: an update. Br J Anaesth. 2013; 110(suppl_1):i53-72.

22. Davidson AJ, Disma N, De Graaff JC, Withington DE, Dorris L, Bell G, Stargatt R, Bellinger DC, Schuster T, Arnup SJ. Neurodevelopmental outcome at 2 years of age after general anaesthesia and awake-regional anaesthesia in infancy (GAS): an international multicentre, randomised controlled trial. Lancet. 2016;387(10015):239-50.

23. Sun LS, Li G, Miller TL, Salorio C, Byrne MW, Bellinger DC, Ing C, Park R, Radcliffe J, Hays SR. Association between a single general anesthesia exposure before age 36 months and neurocognitive outcomes in later childhood. JAMA. 2016;315(21):2312-20.

\section{Publisher's Note}

Springer Nature remains neutral with regard to jurisdictional claims in published maps and institutional affiliations.

\section{Ready to submit your research? Choose BMC and benefit from:}

- fast, convenient online submission

- thorough peer review by experienced researchers in your field

- rapid publication on acceptance

- support for research data, including large and complex data types

- gold Open Access which fosters wider collaboration and increased citations

- maximum visibility for your research: over $100 \mathrm{M}$ website views per year

At $\mathrm{BMC}$, research is always in progress.

Learn more biomedcentral.com/submissions 